\title{
Environmental Condition Monitoring System of Textile Industry for Sustainable Development Goal
}

\author{
Saravanakumar G, Baalachandar C V, Mohaideen Abdul Kadhar K
}

\begin{abstract}
One of the alarming threats to mankind and other living organisms is environmental pollution. Worldwide, textile industry is one of the main air and water pollutants. Its environmental effect is critical because it consumes considerable water for processing and discharges major polluted water. If the quality of those discharged water exceeds the prescribed limit set by the authorities, it will cause serious threat to the living organism. This demands the suitable monitoring system even from a remote location. Innovation in technology could serve as the best solution to the above problem. The chemical properties are measured of the textile effluent and an announcement will be made for proactive measures if it exceeds the prescribed limit. The quality parameters of the effluent are continually monitored and information recorded in the cloud by means of different sensors. The parameters such as $\mathrm{pH}$, and dissolved oxygen of the effluent can be measured and surrounding air quality also measured. The information stored can be tracked by the competent authority via the Web page. The threshold value for the cloud information is automated to make an automatic comparison with the detection system and send an alert to the authorities involved.
\end{abstract}

Keywords: Water Pollution, Air Quality, Sustainable development goals, Raspberry $P i, \quad p H$ measurement, Turbidity measure-ment, Textile industry, IoT.

\section{INTRODUCTION}

Over the years, the quality of clean air and water has declined overall because of various variables, notably industrial waste. The clean water for all is also heavily insisted on the sixth sustainable development goals. Many industries are linked with air and water pollution worldwide, directly and indirectly. The United Nations Environment Programme (UNEP) emphasized that hundreds of million tons of chemical are discharged to the water body [2]. Among the many industries, Textile processing is the considerably major one which supported the conversion of raw material into yarn then into fabric material.

Revised Manuscript Received on December 15, 2019.

* Correspondence Author

Saravanakumar G*, Department of Instrumentation and Control Engineering, Kalasalingam Academy of Research and Education, Srivilliputhur, INDIA - 626121. Email: gurusaravana @ieee.org

Baalachandar C V, Department of Instrumentation and Control Engineering, Kalasalingam Academy of Research and Education, Srivilliputhur, INDIA - 626121. Email: c.v.baala@gmail.com.

Mohaideen Abdul Kadhar K, Department of Electronics and Communication Engineering, Dr Mahalingam College of Engineering and Technology, Pollachi, INDIA - 626121. Email: mohaideen9683@gmail.com.
Finally, fabric is produced after dying or printing. Textile industry not solely includes great quantity of water for the method however additionally want varied chemicals and coloring agents for the process [2] Pollution from textile processing may be in the form of air, water or noise deemed to be environmentally hazardous to health. The textile industry alone consume water of $2.07 \%$. Each time the production method differs based on the customer's need, the waste produced varies based on the type of chemicals and raw materials used. Although these pollutants are inevitable, they can be decreased to some degree and can be discharged which can decrease the environmental effect. The textile industry used natural dyes that did not damage living humans and the environment, but have disappeared these days, when science and technology lagged behind. To accomplish the goal, companies adhere to the use of non-biodegradable synthetic fibers and eventually spoil water and soil resources when discharged without adequate therapy. The rate of water pollution moreover depends on the quantity of water and chemical substances used in the production process [3].

In India, the government is investing more to handle water pollution, but it is sometimes measured in terms of the prevalence rate of a substance in aqueous solution that is below the satisfactory point. Several experiments are accessible to evaluate water purity, such as BOD (BioChemical Oxygen Demand), $\mathrm{pH}$ and solvent dissolved tests.Consumption of textiles comprises different processes such as sizing, thinning and other processes. The big issue occurs when these products are discharged straight into water bodies, which pollute water. This water pollution does not only affect people and households but also impacts aquatic animals. The quality of the waste water is therefore urgently required to be continuously monitored.[4]

Air usually is a mix of several gasses such as nitrogen (79\%), oxygen (20\%) and natural gasses such as argon, carbon-dioxide $(1 \%)$. Since air is a combination, its structure and proposition vary depending on the locations from moment to time. Air pollution is a state in which air is polluted with elevated chemicals, which generally harm the living and cause severe harm to non-living creatures. Recently, the focus has been on controlling emerging problems of dangerous air pollutants, which are mainly exposed in our homes or workplaces where most of our lives is spent.

Published By:

986 Blue Eyes Intelligence Engineering \& Sciences Publication 


\section{Environmental Condition Monitoring System of Textile Industry for Sustainable Development Goal}

The sulphate, which generates sulphur dioxide and fur through the weaving and spinning process, emanates from a polycondensation in textile processing usually. Those parameters which affect the environment are therefore necessary to measure [5], [6].

A range of water quality control systems across the country have been established by the CPCB (Central Pollution Control Board) that monitor water quality on a monthly or annual basis. This is done to ensure that the quality of water is maintained or not maintained. It is essential to be regularly monitored. The nature of the pollution control requirements and the efficiency of pollution controls are assessed by water quality and air quality surveillance. Although various protocols and rules for efficient effluent disposal have been drawn up by the Government, there always are violations for the business interests of the sector.[7]

\section{PROBLEM STATEMENT AND RELATED WORK}

Water and air pollution in the textile industry increases every day. Government authorities often visit sectors to monitor the quality parameter. However, afterwards, any time industries can release damaging waste that contaminates both water and air. There is therefore a need for a mechanism to remotely monitor the quality parameter of the bodies. The suggested scheme will allow the authorities to remotely monitor the parameters by putting the surveillance system, especially at their point of discharge. The environmental impact of textile effluents is evaluated by many researchers and scientists. Many researchers and scientists are analyzed the impact of textile effluent to the environment. In [6], Kant pointed out that synthetic dyes are harmful to environment and people which uses the complex chemicals. He alerted that, In 2020, more than $60 \%$ of people allergic to chemical and demanded the positive action to alleviate the problem. Environmental risk analysis in textile industry was studied in [5]. They pointed out the risk and hazards due to effluent that discharged to the local water bodies and also gas emission and related odor generation while processing. The parameters of water quality are evaluated with distinct methods due to advances in technology. The water quality parameters are measured and transmitted to distant place by using Zigbee and intimated to the officials by SMS using GSM which was reported in [8]. They proposed the solution to conventional method of measurement. Microcontroller based remote environmental monitoring system is developed in [9] . They have measured the $\mathrm{pH}$, Temperature, Relative humidity and volatile organic components of water. The measured data shared via cloud platform. The smart wireless sensor nodes are used to collect the parameters related to municipal drinking water quality. Further, the complex algorithms are used to detect the quality of water[10]. The low-cost intelligent system for air and water quality monitoring system was developed in [8]. They utilized the microcontroller-based data acquisition system and transmitted the date to the cloud for storage.[11], [4].

\section{SYSTEM DESIGN}

Our aim is to create a real-time air and water quality assessment for the textile industry from a distant place with Raspberry $\mathrm{Pi}$ (RPi). The comprehensive block diagram is shown in the Figure. 1. In Arduino Uno, where a sensor is connected, it acquires a real-time water and air parameter. In order to gain the required parameters in real time for monitoring water health, $\mathrm{pH}$ and turbidity sensors are

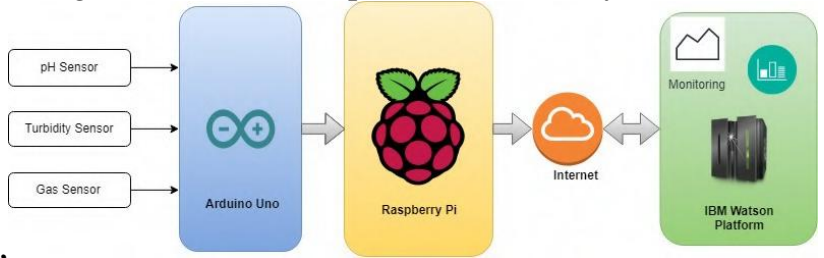

Fig. 1. Block Diagram

TABLE

I

HA R DWA RE A ND SOF

TWA RE TOOL

\begin{tabular}{|l|l|}
\hline HARDWARE & SOFTWARE TOOL \\
\hline Raspberry pi 3 & Raspbian JESSIE \\
Arduino & Arduino \\
UNO & IDE Node- \\
Turbidity & RED \\
sensor & Firmata \\
\hline
\end{tabular}

and air quality sensors are used to collect information to monitor air quality in the real time. The following hardware and software are utilized for the development of the proposed system as given in Table. I

\section{A. Hardware design/Configuration}

1) Raspberry Pi: The Raspberry Pi 3 Model B is a great platform for building automation systems. Rpi 3 Model B is a computer that is small and is able to perform the whole work of an ordinary desktop computer. It is a core of the system, used especially for communication with cloud and web applications. The BCM 2837 64bit ARM v8 processor, on board BCM 43438 Wi-Fi, 1 GB RAM,4x USB 2 ports, 40 pin GPIO expanded, HDMI and RCA video output comprises of a 1,2 GhZ QUAD Core Broadcom processor. It has a distinct slot to store the operating system and the other necessary software and controllers for the Micro SD cards slot. Raspberry Pi is able to support various operating systems, like Raspbian, Windows 10 or Ubuntu, etc. Raspbian Jessie will be loaded and operated [21], [28], [29]. 


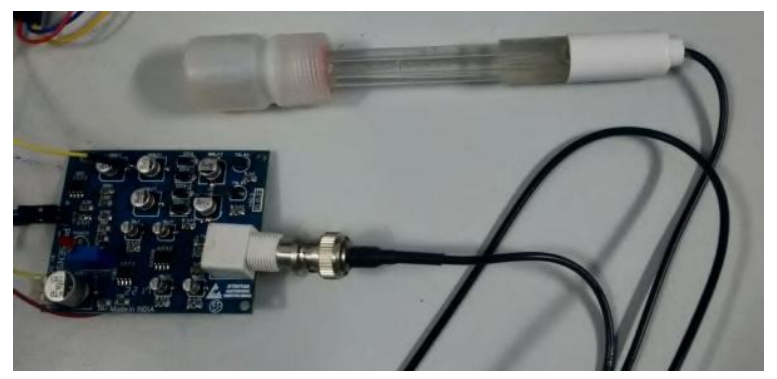

Fig. 2. pH Sensor

2) Arduino Uno: Arduino is a microcontroller platform open source to develop real-world data acquisition and control system. The board is fitted with sets of input / output (I / O) digital and analog buttons, which can be interfaced with several controllers and other systems[11]. The panel consists of 14 digital pins, 6 analog pins, and can be programmed via

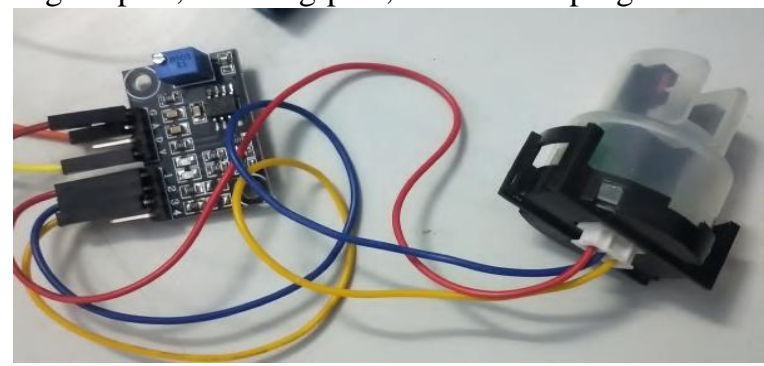

Fig. 3. Turbidity

Sensor

an Arduino IDE via an USB cable of type B. Powered by an USB cable or an internal 9 Volt battery, it accepts voltages from 7 to 20 Volts. In order to gather the physical parameters of water and air quality, the sensor is all linked to the Arduino board.

3) $\mathrm{pH}$ Sensor and Probe: The measurement of hydrogen component concentration in the fluid is $\mathrm{pH}$ value $\mathrm{PH}$ measurements are mainly made with $\mathrm{pH}$-sensitive glass electrodes that generally have proven satisfactory for measurements of $\mathrm{pH}$. Changes in fluid junction potential between the test solution and the standardizing buffer should ideally be low or at least extremely reproducible. The $\mathrm{pH}$ of a solution is the measure of its acidity or alkalinity. The $\mathrm{pH}$ is a logarithmic scale with a range of 0-14 and a neutral point of 7 . Values above 7 show an acidic solution, fundamental or alkaline, and levels below 7 are indicated. Values above 7 indicate a basic or alkaline solution and values below 7 would indicate an acidic solution. It operates on $5 \mathrm{~V}$ power supply and it is easy to interface with Arduino.

The electrode, the glass membrane and the container are part of a $\mathrm{pH}$ probe. It has a simple, convenient and practical connection and features. The $\mathrm{pH}$-circuit and circuit connection of the BNC connector can be read readily into any analog input of microcontroller.

The $\mathrm{pH}$ probe consists of two main components, such as a measuring electrode made up of a glass electrode and also referred to as a skin or active electrode and a reference electrode receiving the provided voltage, a reference electrode and a normal electrode.

4) Turbidity Sensor and Probe: Turbidity is described as reducing a liquid's transparency induced by the appearance of unresolved suspended material. It blocks out the light that overflows with water. It can also increase above normal surface water temperatures as suspended particles close the surface facilitate heat absorption from sunlight. Mineral or organic may be the source of the particles discovered in waste water. However, turbidity is not a direct measure of suspended particles in water, but a measure of the scattering impact

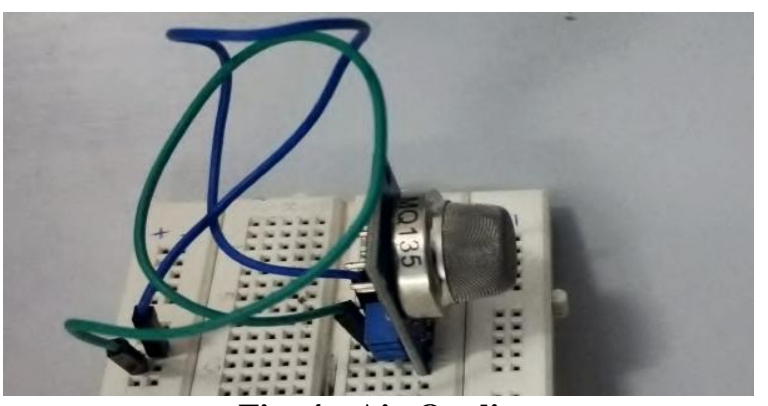

Fig. 4. Air Quality sensor(MQ135)

on light of these particles. Sensors of turbidity evaluate the quantity of light dispersed in water by the suspended solids. As the amount of total suspended solids (TSS) in water increases, the water's turbidity level increases. Turbidity probe is made of plastic covered circuit. Inside the housing, it contains a probe circuit which includes photo emitting diode and photo transistor which receives the light. It is made waterproof so that the water does not move inside the housing. The amount of light received determines the turbidity of water. As the particles in the water increases, the amount of light transmitted decreases, which helps us to measure the amount of turbidity in water.

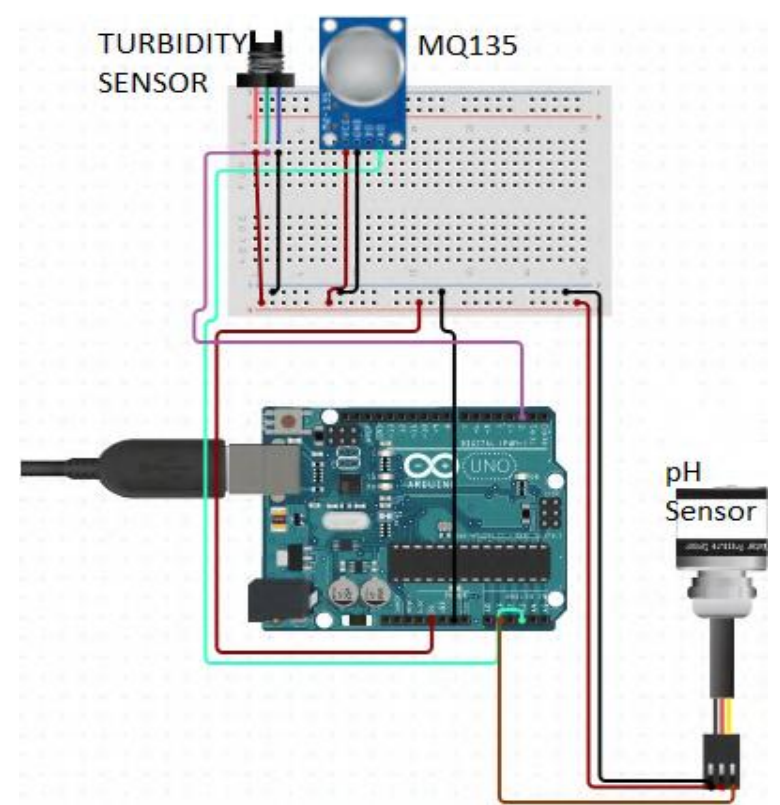

Fig. 5. Schematic diagram

Published By:

988 Blue Eyes Intelligence Engineering \& Sciences Publication 


\section{Environmental Condition Monitoring System of Textile Industry for Sustainable Development Goal}

5) Air Quality Sensor (MQ-135): The MQ-135 gas sensor senses the gases like ammonia nitrogen, oxygen, The Gas Sensor MQ-135 senses the gasses of ammonia such as nitrogen, oxygen, alcohol, sulphide and smoke. The MQ-3 chip gas sensor boost converter is PT1301. This gas sensor has a working voltage between $2.5 \mathrm{~V}$ and $5.0 \mathrm{~V}$. To detect smoke, benzene, steam, and other damaging gases, the MQ-135 gas sensor can be introduced. It can detect various damaging gasses. The gas detector MQ-135 is cheap to buy. Figure 4 displays the fundamental picture of the sensor.

B. Software design/Configuration

1) Raspbian Jessie: Raspbian Jesie is a Linux-based open source operating system that can be loaded to the Raspberry Pi. You can load it from raspberrypi.org/downloads/ and use the Etcher or NOOBS package to burn it into the SD card [26].

2) Arduino IDE: The Arduino Integrated Environment Development (IDE) is a cross-platform application written in the language of Java programming [22], [27]. It is used for writing and downloading programs to boards compatible with Arduino, but also for other developer boards with the assistance of third-party cores. The RPi / Arduino communication takes place via the Firmata, one of the communication protocols for establishing communication between microcontrollers and computers via the software [23]. Standard Firmata is a library of software that lets devices from Arduino communicate using the Firmata protocol with your PC or Raspberry Pi. For beginning the outline and the primary program loop, the user code needs only two fundamental features. The Uno board is the first in a range of USB Arduino boards and the Arduino platform reference model. An input or output can be used for each of the 14 digital pins and 6 Uno analog pins.

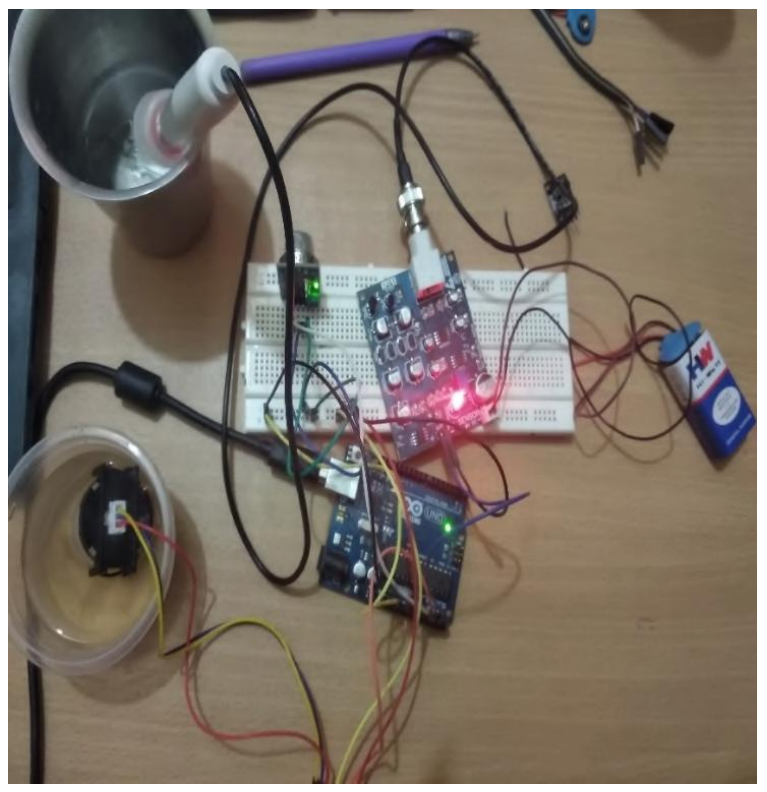

Fig. 6. Prototype connections

4) IBM Watson IoT Platform: It's the Cloud based service for IoT devices for IBM Watson IoT platform. In order to gain valuable information, sensor information can be stored and analyzed [17].

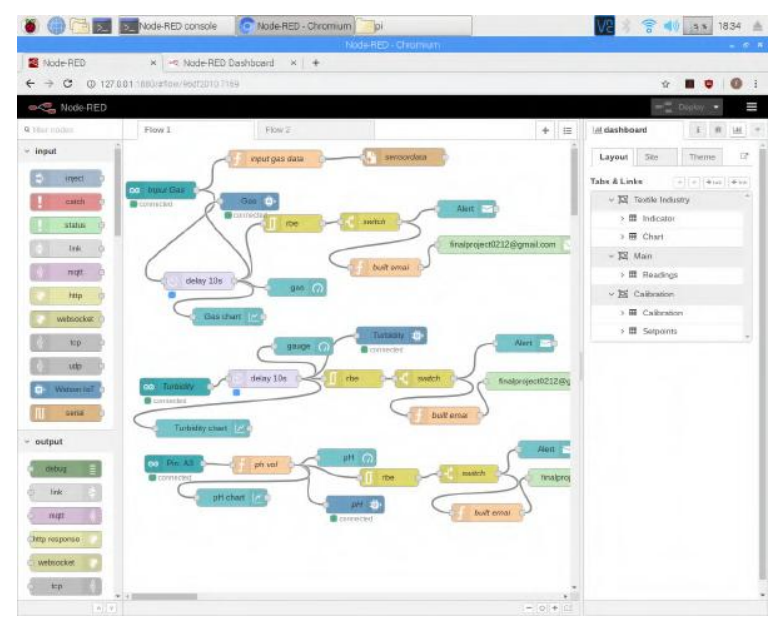

Fig. 8. Node-RED

Program

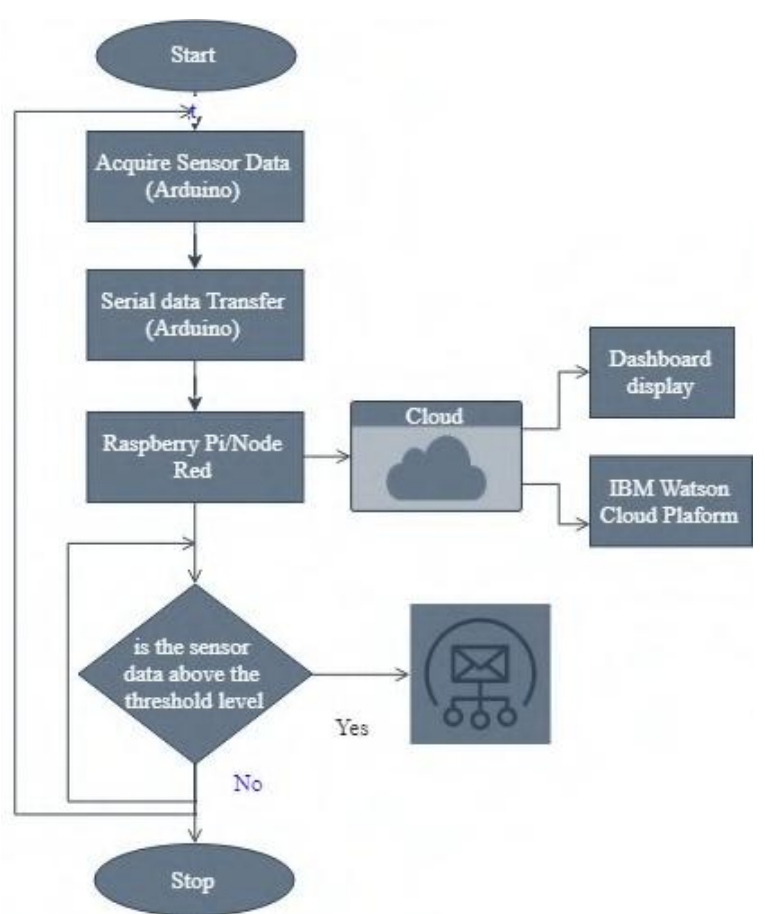

Fig. 7. Flow

Chart

3) Node-RED: Node-RED is a instrument for IoT apps, which can be used easily, fundamentally and open source. This visual programming tool is extremely used, which helps IoT developers incorporate hardware, APIs and on-line services in a very exciting and creative way. The Node-Red library consists of thousands of flows and nodes to allow the user to connect all manner of devices and services. Flows can be run on hardware such as Raspberry pi or in a cloud on the network's edge since node-red runtime includes node.js. NodeRed offers a single click mechanism to use a light weight runtime environment to deploy the IoT developer's flows [14]. 


\section{HARDWARE AND SOFTWARE IMPLEMENTATION}

The hardware connections are established as per the Fig. 5 and software configuration are made as per the details given in the previous section. The physical connection of the hardware prototype is shown in Fig.6. Rpi and Arduino serves as the heart of this module which controls the entire process. After all the initialization and configuration for both software and hardware. Firstly, the sensors are connected to the Arduino of respective pins. Communication was established between Arduino and Rpi by using Standard Firmata protocol.

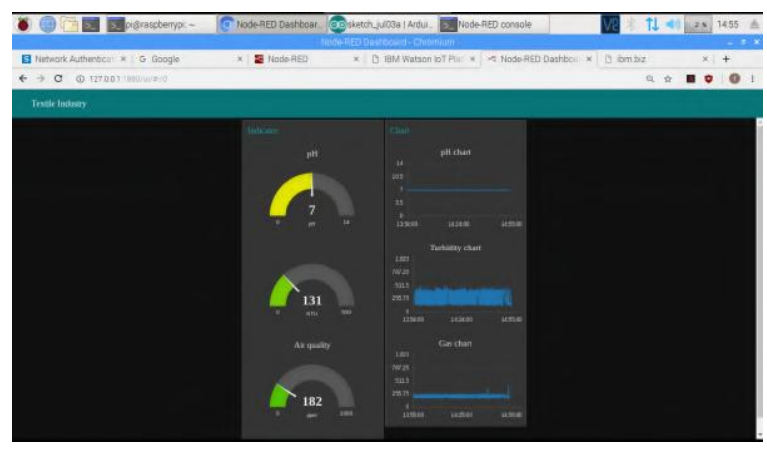

Fig. 9. Node-RED

Dashboard

Node-RED is a graphical programming environment where program is developed for integrating the sensors. Firstly, The Arduino nodes are created to sense the air and water quality data. The sensor data is compared with the threshold set by the authorities [19]. The air quality, $\mathrm{pH}$ and Turbidity sensor given to the Arduino which acts as an analog to digital converter and the Arduino communicate with the raspberry pi using USB Serial communication and from Raspberry Pi the program is done in Node-RED which collects the sensor data from the Arduino. The threshold value is set in the program of the node red for Turbidity sensor as (300-500 NTU), pH sensor as (6.58) and Gas sensor as (500 ppm). Further, the nodes are created to alert the authorities through email. If the threshold reaches the limit, then automatically an alert message is sent through eMail. Meanwhile, the nodes are created to communicate with IOT Watson Platform. Finally, the Dashboard is configured to display the sensed parameters in the web page. The created dashboard is shown in Fig. 9. The values will be displayed in the Node-Red dashboard. The measured values will also be stored in the IBM Watson an IoT platform. The detailed programming flow is given in Fig. 8 [18].

\section{RESULTS AND DISCUSSION}

Dashboard is the clear visual indication of the sensor data. The $\mathrm{pH}$ value, turbidity value and air quality values are displayed in both dial and trend format. Node-RED dashboard can have varied feature to visualize the data in different form. The user can locally or remotely visualize this dashboard which is given in Fig. 9. Otherwise through the dedicated web page of IBM Watson IoT platform, the users/authorities can have access to the sensor reading and can see real-time air and water quality

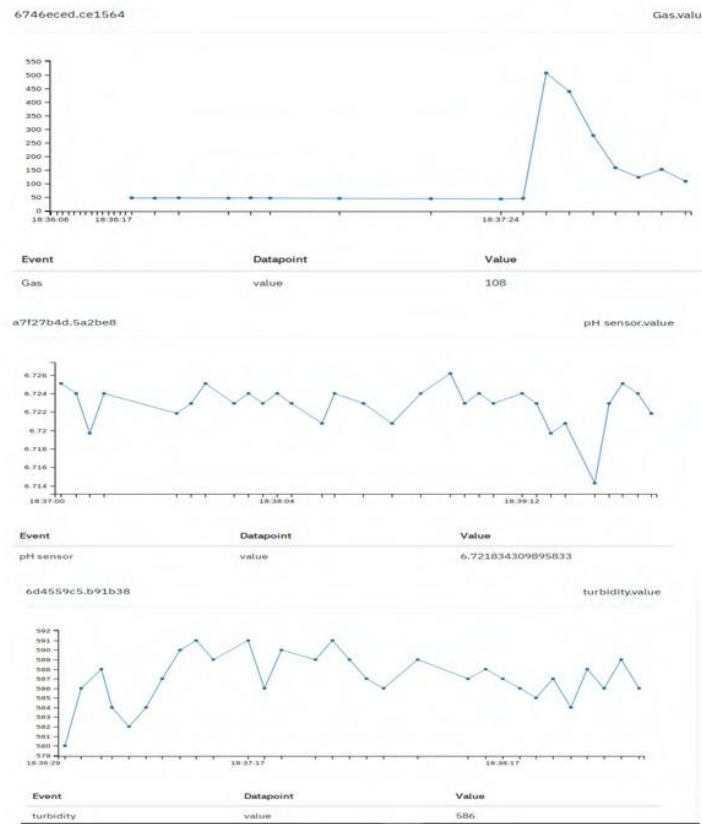

Fig. 10. sample of Sensor Data in IBM Watson IoT Platform

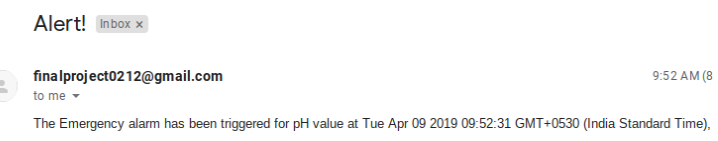

Fig. 11. Sample eMail Alert system

parameters that are stored in cloud service. The data plotted in IBM Watson is shown in Fig.10. The threshold value may be adjusted by the authorities based on the different standards. The alert can be emailed to various layer officials. The sample alert system is shown in Fig.11. Authorities can decide to solve this problem constructively in order to reduce water and air contamination.

\section{CONCLUSION AND FUTURE SCOPE}

Each organization should regularly monitor effluents and documents related to the environmental safety regulations in order to protect the environment. The system is developed which can remotely monitor the release by the textile processing industry of toxic gases and water effluent. The level of pollution is emitted by industry is monitored using Rpi and IBM Watson IoT platform which can prevent environmental pollution and safety to the living beings. Air Quality (MQ135) sensors detect the poisonous gases. The turbidity and $\mathrm{pH}$ sensors monitor the quality of water effluent from the textile industry and alert can be given to save the life of people and for the environment. Remote monitoring is enabled by using IBM Watson IoT platform. The application on Cloud shows data collected from the sensors.
Blue Eyes Intelligence Engineering \& Sciences Publication 


\section{Environmental Condition Monitoring System of Textile Industry for Sustainable Development Goal}

The stored data are compared with threshold of standard. In the case of deviation, it alerts the authorities via email.

Thus, device helps us to identify the pollution from the textile processing industry. The developed system is an initiative to the sustainable development goals to reduce the air and water pollution for clean water for all. In future, the qualitative parameters can be evaluated by extra sensors.

\section{REFERENCES}

1. U. Nations, The sustainable development goals report 2016, 2019.

2. I. United Nations Environment Programme. Division of Technology, Economics, E. Programme, and United Nations Environment Programme [UNEP], "UNEP Year Book 2014: Emerging Issues in Our Global Environment," Emerging issues in our global environment, 2014.

3. K. Saravanan, E. Anusuya, R. Kumar, and Others, "Real-time water quality monitoring using Internet of Things in SCADA," Environmental monitoring and assessment, vol. 190, no. 9, p. 556, 2018.

4. M. S. Islam, "An Intelligent System on Environment Quality Remote Monitoring and Cloud Data Logging Using Internet of Things (IoT)," International Conference on Computer, Communication, Chemical, Material and Electronic Engineering, IC4ME2 2018, pp. 1-4, 2018.

5. V. Jaganathan, P. Cherurveettil, A. Chellasamy, and M. S. Premapriya "Environmental pollution risk analysis and management in textile industry: A preventive mechanism," European Scientific Journal, ESJ, vol. 10, no. $10,2014$.

6. R. Kant, "Textile dyeing industry an environmental hazard," Natural science, vol. 4, no. 1, pp. 22-26, 2012.

7. F. Ezbakhe, "Addressing Water Pollution as a Means to Achieving the Sustainable Development Goals," Journal of Water Pollution and Control, vol. 1, no. 1:6, pp. 1-9, 2018.

8. M. Shirode, M. Adaling, J. Biradar, and T. Mate, "IOT Based Water Quality Monitoring System," International Journal of Computer Sciences and Engineering, vol. 12, pp. 5447-5454, 2018.

9. N. A. Cloete, R. Malekian, and L. Nair, "Design of smart sensors for real-time water quality monitoring," IEEE Access, vol. 4, pp. 3975-3990, 2016.

10. S. Kavi Priya, G. Shenbagalakshmi, and T. Revathi, "Design of smart sensors for real time drinking water quality monitoring and contamination detection in water distributed mains," International Journal of Engineering \& Technology, vol. 7, no. 1.1, p. 47, 2017.

11. B. Das and P. C. Jain, "Real-time water quality monitoring system using Internet of Things," in 2017 International Conference on Computer, Communications and Electronics (Comptelix). IEEE, 2017, pp. 7882.

12. S. Geetha and S. Gouthami, "Internet of things enabled real time water quality monitoring system," Smart Water, vol. 2, no. 1, p. 1, 2016.

13. M. Simić, G. M. Stojanović, L. Manjakkal, and K. Zaraska, "Multisensor system for remote environmental (air and water) quality monitoring," in 2016 24th Telecommunications Forum (TELFOR). IEEE, 2016, pp. 1-4.

14. M. Lekić and G. Gardašević, "IoT sensor integration to Node-RED platform," 2018 17th International Symposium on INFOTEH-JAHORINA, INFOTEH 2018 - Proceedings, vol. 2018-Janua, no. March, pp. 1-5, 2018.

15. Y.-T. Liu, B.-Y. Lin, X.-F. Yue, Z.-X. Cai, Z.-X. Yang, W.-H. Liu, S.Y. Huang, J.-L. Lu, J.-W. Peng, and J.-Y. Chen, "A solar powered long range real-time water quality monitoring system by LoRaWAN," in 2018 27th Wireless and Optical Communication Conference (WOCC). IEEE, 2018, pp. 1-2.

16. K. H. Kamaludin and W. Ismail, "Water quality monitoring with internet of things (IoT)," in 2017 IEEE Conference on Systems, Process and Control (ICSPC). IEEE, 2017, pp. 18-23.

17. Ibm, "IBM IoT," 2016.

18. S. Monk, Raspberry Pi Cookbook, 2016.

19. T. P. C. Board, "A READY RECKONER FOR ENTREPRENEURS," 2017.

20. W.-C. Lin, K. Brondum, C. Monroe, and M. Burns, "Multifunctional water sensors for $\mathrm{pH}, \mathrm{ORP}$, and conductivity using only microfabricated platinum electrodes," Sensors, vol. 17, no. 7, p. 1655, 2017.

21. Raspberry Pi Foundation, "Raspbian," 2018.

22. D. Wheat and D. Wheat, "Arduino Software," in Arduino Internals, 2012.
23. H.-c. Steiner, "Firmata: Towards making microcontrollers act like extensions of the computer," New Interfaces for Musical Expression, 2009.

24. IBM, "Internet of Things Platform - IBM Cloud," 2018.

25. Raspberry Pi Foundation, "Raspberry Pi 3 Model B," Raspberry Pi, 2016.

26. Eben, "Download NOOBS for Raspberry Pi," 2008

27. Arduino, "Software Arduino (IDE)," 2017.

28. S. McManus, "Raspberry Pi For Dummies," in John Wiley \& Sons, Inc, 2014.

29. Raspberry Pi Foundation, "Raspberry Pi 3 Model B+ Datasheet," Datasheet, 2016.

30. A. Rajalakshmi and H. Shahnasser, "Internet of things using node-red and alexa," in 2017 17th International Symposium on Communications and Information Technologies, ISCIT 2017, 2018.

31. M. Tabaa, B. Chouri, S. Saadaoui, and K. Alami, "Industrial Communication based on Modbus and Node-RED," in Procedia Computer Science, 2018. 